\title{
Design of High-Average-Power Near-Millimeter Free Electron Laser Oscillators Using Short-Period Wigglers and Sheet Electron Beams
}

JOHN H. BOOSKE, MEMBER, IEEE, DANIEL J. RADACK, THOMAS M. ANTONSEN, JR., MEMBER, IFEE, STEVEN W. BIDWELL, YUVAL CARMEL, MEMBER, IEEE, WILlIAM W. DESTLER, MEMBER, IEEe, HENRY P. FREUND, VICTOR L. GRANATSTEIN, SENIOR MEMBER, IEEE, PETER E. LATHAM, MEMBER, IEEE, BARUCH LEVUSH, MEMBER, IEEE, ISAAK D. MAYERGOYZ, FELLOW, IEEE, AND A. SERBETO

\begin{abstract}
The design and feasibility of a 1-MW continuous wave (CW) free electron laser (FEL) oscillator are reviewed. The proposed configuration will include a short-period $\left(l_{n}-1 \mathrm{~cm}\right)$ planar wiggler, a sheet electron beam, a 0.5-1.0 MV thermionic electron gun, a hybrid waveguide/quasi-optical resonator, commercial dc power supplies, and a depressed collector. Cavity ohmic RF losses are estimated to be extremely low ( $\leqslant 10-100 \mathrm{~W} / \mathrm{cm}^{2}$ ) at 1-MW output power, while thermal heat transfer studies conservatively indicate that wall cooling up to 1500 $W / \mathrm{cm}^{2}$ should be possible. Experiments have convincingly verified theory and simulations which predict that negligible body currents will be achievable with low-emittance low-space-charge sheet beams. Highvoltage sheet beam gun design studies indicate that the required beam quality can be achieved with $\mathrm{CW}$ compatible devices. The spent beam energy distribution is consistent with highly efficient spent beam energy recovery, and the proposed resonator cavity should provide mode discrimination and beam/RF separation capability. Finally, recent advances in superconducting wiggler designs suggest that even more compelling improvements in FEL design are possible. Specifically, the substantially increased dc wiggler fields can yield better beam confinement. Furthermore, options for a strong-pump FEL amplifier with a tapered, superconducting, short-period wiggler are now being considered.
\end{abstract}

\begin{tabular}{|c|c|}
\hline Nomer & CLATURE \\
\hline$V_{\text {beam }}$ & Beam voltage. \\
\hline$\gamma=1+\left(e V_{\text {beam }}\right)\left(m c^{2}\right)$ & $\begin{array}{l}\text { Relativistic energy parame- } \\
\text { ter. }\end{array}$ \\
\hline$I_{\text {beam }}$ & Beam current. \\
\hline$b_{\text {beam }}$ & $\begin{array}{l}\text { Small transverse equilib- } \\
\text { rium dimension of the } \\
\text { sheet electron beam in the } \\
\text { cavity. }\end{array}$ \\
\hline$a_{\text {beam }}$ & $\begin{array}{l}\text { Wide transverse dimension } \\
\text { of the beam. }\end{array}$ \\
\hline
\end{tabular}

Manuscript received October 25, 1989; revised December 12, 1989. This work was partially supported by the U.S. Department of Energy, the ONR, and by the SDIO/IST through a contract administered by Harry Diamond Laboratories.

J. H. Booske is with the Department of Electrical and Computer Engineering, University of Wisconsin, Madison, WI 53706.

D. J. Radack, T. M. Antonsen Jr., S. W. Bidwell, Y. Carmel, W. W Destler, V. L. Granatstein, P. E. Latham, B. Levush, I. D. Mayergoyz, and $A$. Serbeto are with the Laboratory for Plasma Research, University of Maryland, College Park, MD 20742.

H. P. Freund is with Science Applications International Corporation, McLean, VA 22102.

IEEE Log Number 9035368 $\theta_{\max }$

$b_{r f}$

$a_{r f}$

$T_{\text {eff }}$

eff

$L$

$l_{w}$

$B_{\mathrm{w}}$.

$\lambda_{\beta}$

$f$

$\theta_{\text {intercept }}$

$\hat{P}_{\text {wall }}^{r f}$

$P_{\text {cav }}$

$P_{\text {out }}$
Maximum divergence angle allowed for electron injected at beam edge. An edge electron with this divergence angle will have a maximum betatron orbit amplitude of $\mathcal{Y}_{\beta}=$ $b_{\text {beam }} / 2$.

Narrow transverse waveguide dimension.

Wide transverse waveguide dimension.

Net transmissivity coefficient includes both diffractively coupled output power and ohmic wall losses.

Wiggler length.

Wiggler period.

Number of wiggler periods.

Peak on-axis amplitude of wiggler field.

Betatron wavelength for betatron orbits in the narrow ( $y$ ) transverse dimension.

Radiation frequency.

Electronic, or intrinsic, FEL interaction efficiency.

Injected divergence angle at which an edge electron will intercept the cavity wall. This estimate is based on the somewhat overconservative formula of (4b).

RF ohmic wall losses in watts per square centimeter.

Circulating cavity power.

Diffractively coupled output radiation power. 


\section{INTRODUCTION}

$\mathrm{T}$ HE RESULTS described in this paper constitute the status of research on free electron lasers (FEL's) at the University of Maryland. The focus of the overall program is to develop a short-period-wiggler (SPW) free electron laser (FEL) with a sheet electron beam as a source of high-average-power millimeter-wave radiation for electron cyclotron resonance heating $(\mathrm{ECRH})$ in advanced magnetic fusion experiments such as the Compact Ignition Torus. Other applications include sources for nearmillimeter space-based radar.

The general specifications [1] for the ECRH sources call for 10-30 MW of average power with pulse durations of several seconds to $\mathrm{CW}$. The radiation frequency should be near $300 \mathrm{GHz}$, with possible interest in $-600 \mathrm{GHz}$ for second-harmonic ECRH. A premium is placed on high system efficiency, as well as the utilization of established (i.e., low-risk), off-the-shelf technology where possible. Similarly, there is a demand for high system reliability and flexibility in future utilization of the ECRH source(s). Finally, to ensure experimental physics flexibility, it is desirable that the radiation source have $\sim 30 \%$ total operating bandwidth with $\sim 5 \%$ rapid dynamic bandwidth (timescale $\leqslant 10 \mathrm{~ms}$ ).

One proposed solution to this need is an FEL CW oscillator which uses:

1) a short period $\left(l_{w} \sim 1 \mathrm{~cm}\right)$ planar wiggler,

2) a "low" voltage ( $V_{\text {beam }} \leqslant 1 \mathrm{MV}$ ) sheet electron beam generated by a $\mathrm{CW}$, electrostatic, thermionic Pierce gun,

3) a depressed collector for spent beam energy recovery, and

4) two dc, "off-the-shelf", high-voltage power supplies; one bias supply at $0.5-1.0 \mathrm{MV}$ drawing $\leqslant 100$ $\mathrm{mA}$, and a second supply at $<200 \mathrm{kV}$, providing $\sim 30-60 \mathrm{~A}$ of beam current.

A simplified schematic of the basic configuration is shown in Fig. 1. The logic supporting the preceding choices is reviewed next.

The choice of an FEL is based on the strong established experimental database demonstrating high-power highfrequency operation at parameters relevant to the needs for ECRH. Natural wideband frequency tunability and low RF wall losses are among other compelling features of conventional FEL's. Our proposed use of CW operation

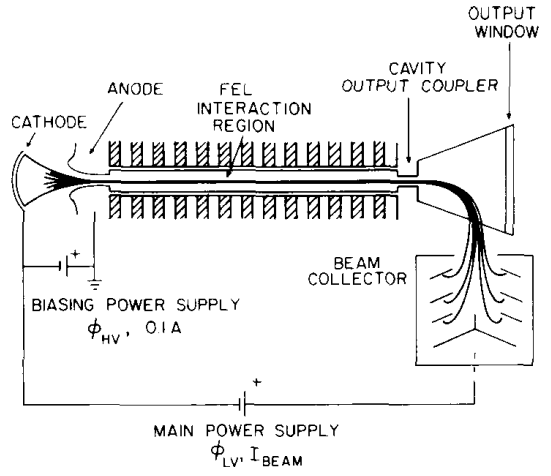

Fig. 1. Simplified conceptual layout and circuit diagram for sheet beam FEL oscillator, including spent beam energy recovery.

is intended to avoid hysteritic cyclic stress failure in critical system components. Cyclic fatigue is a common failure mode in repetitively pulsed high-power mechanical and electronic systems. Cavity cracking in recent highpower gyrotrons at Varian [2] is just one more-very germane-example of this problem.

By specifying a planar wiggler with short spatial period $\left(l_{w} \sim 1 \mathrm{~cm}\right)$ one reduces the requirement on beam voltage ( $V_{\text {beam }} \leqslant 1 \mathrm{MeV}$ ) to achieve millimeter-wave ( $\geq 300$ $\mathrm{GHz}$ ) operation [3], [4]. At these voltages, one can generate the required beam power with established off-theshelf dc power supply technology. Hence in this regard, the issue is power supply cost rather than the risk of developing new technology. These voltages are also compatible with the design of $\mathrm{CW}$ electrostatically focused thermionic Pierce guns. The short period wiggler also yields a smaller total size, reducing magnet cost and complexity and generally making it possible to package the advantages of conventional FEL's in a "compact," transportable, and commercializable "tube technology."

High-power radiation output necessitates high beam power. To obtain this capability without invoking high current density, we have proposed the use of a sheet or ribbon electron beam [5]. Such beams are naturally suited to stable and well-focused transport in planar wigglers when an axial magnetic guide field is not used [6]. In addition, a streaming beam without an axial guide field is easier to generate and match to an interaction region than rotating beams with axial fields.

Spent beam energy recovery of streaming beams with small exit energy spread should be considerably easier than recovery of rotating beams with large exit energy spread. The issues here involve size and complexity of the depressed collector region and associated power supplies. Rotating beams must first be "unwound" in a magnetic field taper before energy recovery can be effectively attempted. At modest beam voltage $(\sim 100 \mathrm{kV})$ this may not be a severe constraint, but at higher beam voltages ( $V_{\text {beam }} \geq 500 \mathrm{kV}$ ) the taper length may become considerable. Streaming beams, however, require no such "beam conditioning." The exit energy spectrum of the 
beam determines the size and complexity of an efficient depressed collector. Experimental experience has demonstrated that collector efficiencies in excess of $90 \%$ are possible for low-gain FEL oscillators with beam voltages of 1-6 MV [7]-[9]. Simulations suggest that such capability may be possible for the FEL proposed here with a minimal number of electrodes in the depressed collector. If true, this would not only yield total FEL system efficiencies of $-30-40 \%$, but would allow the majority of the beam current to be drawn from less expensive lowvoltage $(V \leqslant 200 \mathrm{kV})$ power supplies.

It is noted that the underlying concept of a CW FEL oscillator with depressed collectors is not new to this paper, but has been previously proposed in [10]. Features of this work which differ from the previous study [10] include the sheet beam, short period wiggler, lower beam voltage, a grounded tube body, the use of conventional dc power supplies, and the generation of the beam with a thermionic Pierce gun.

To evaluate the feasibility of a $\mathrm{CW}$ high-power millimeter-wave source, we have identified the principal technological risk issues for the short-period-wiggler sheet beam FEL as

1) thermal engineering,

2) beam transport,

3) beam generation,

4) spent beam energy recovery,

5) power supply technology,

6) magnet technology, and

7) cavity design.

The remainder of this paper discusses our program's progress in assessing the risks associated with each of these topics.

\section{Self-Consistent Conceptual Design}

Self-consistent conceptual design of low-gain FEL oscillators has been reduced to a powerful, convenient procedure through the identification of fundamental "universal" quantities [11]-[16]. The technique is described in detail in [11] and [12] and is built upon the analytic and numerical formalisms developed in [11]-[17]. The formulation and codes have been benchmarked against several experiments. First, the time-dependent multimode oscillator codes were used to analyze results from the Hughes Mark-II FEL oscillator [18]. Good agreement was found between theory and experiment for such characteristics as multimode behavior, saturated efficiency, oscillator priming, and slewed voltage effects [19]. A second comparison [15], [20] with the University of California at Santa Barbara FEL [7], [9] has yielded excellent agreement in explaining observations of both single and multimode operation [21] in that device. Finally, when run in a single-pass amplifier mode, the codes have yielded excellent agreement [22] in predicting the single-pass efficiency of the untapered section of the ELF FEL facility at the Lawrence Livermore National Laboratory [23].
To summarize, the universal formalism makes it possible to identify a unique dimensionless operating point with optimized efficiency for low-gain, untapered, Compton, FEL oscillators. This "point" can be transformed into (dimensionalized) device designs through the use of scaling relations which relate the universal quantities to physical observables. With these formulas, one can rapidly modify designs to optimize RF wall losses, output power, etc. while maintaining optimal FEL efficiency. Note that the dimensionless universal formalism has drastically reduced the theoretical parameter space, thereby permitting self-consistent comprehensive investigations of such realistic effects as multimode competition, finite beam turn-on time, dc space-charge, beam energy spread, and wiggler tapering. Most of these effects are discussed in detail in [11]-[17]. The remainder will be described in future publications.

As a general rule, the designs described in this paper are characterized by short interaction lengths, relatively weak wiggler parameters (and thus small transverse beam energy), and low beam current densities. Consequently, the FEL interaction physics are relatively insensitive to beam energy spread $(\Delta \gamma /(\gamma-1)< \pm 2 \%$ is acceptable-see [11]) and beam displacement effects. In addition, for the designs reported here, the use of a sheet beam does not significantly alter the basic physics to the FEL interaction (when compared to a round beam) other than to substantially reduce the de space-charge fields [17]. At beam current densities much higher than prescribed for the designs of this paper, new effects, such as self-focusing in the wide beam transverse dimension, may become important [17]. As examples of what our design method yields, we have provided four untapered $\sim 300 \mathrm{GHz}, \sim 1$ MW FEL oscillator designs in Table I (the symbols of Table I have been defined in the Nomenclature). The fundamental variable in these four designs is the beam voltage, which ranges from 0.5-1.0 MV. To keep the frequency constant as beam voltage increases, we have simultaneously increased the wiggler period length. We will refer to details of these designs throughout the report. Hence, at this point, general observations will be made. First, the estimated RF wall losses are considerably lower in all four designs than for the conventional gyrotron [24] or the (smooth-walled) CARM [25]. At the higher voltages, these wall losses $\left[\hat{P}_{\text {wall }}^{r f}\right]$ are essentially negligible. Note that to compute this ohmic dissipation, a conservative wall conductivity value two times lower than the text book value [26] was used. The second point is that for FEL beam voltages exceeding $600 \mathrm{kV}$, beam-to-wall clearances are comparable to those proposed in $300-\mathrm{GHz}$ whispering gallery gyrotrons [24] and CARM's [25]. This second observation is intended for reference purposes only, and does not imply any inherent advantage per se for the FEL over the gyrotron or the CARM. Previous sheet beam FEL designs using short period wigglers [3][5] proposed parameters similar to the first column of Table I wherein the beam-to-wall clearances were probably impractical for the available beam focusing capability. 
TABLE I

1-MW 300-GHz UNTaPe:Rtid FEL Oscillator Desigins

\begin{tabular}{|c|c|c|c|c|}
\hline$\gamma$ & 1.98 & 2.27 & 2.66 & 2.96 \\
\hline$V_{\text {be:an! }}(\mathrm{kV})$ & 500 & 650 & 850 & 1000 \\
\hline$I_{\text {texim }}(\mathrm{A})$ & 60 & 48 & 40 & 34 \\
\hline$b_{\mathrm{hc}_{\mathrm{c}, \mathrm{II}}}(\mathrm{cm})^{n}$ & 0.18 & 0.20 & 0.24 & 0.26 \\
\hline$a_{h_{v}: m}(\mathrm{~cm})$ & 4.0 & 4.0 & 4.0 & 4.0 \\
\hline$\theta_{\text {mix }}(\operatorname{deg})$ & \pm 2.0 & \pm 2.0 & \pm 2.0 & \pm 2.0 \\
\hline$b_{r}(\mathrm{~cm})$ & 0.22 & 0.375 & 0.575 & 0.70 \\
\hline$a_{r y}(\mathrm{~cm})$ & 5.0 & 5.5 & 6.0 & 6.0 \\
\hline$T_{\text {ifl }}$ (net transmissity) & 0.19 & 0.13 & 0.11 & 0.12 \\
\hline$L(\mathrm{~cm})$ & 16.2 & 19.6 & 25 & 30 \\
\hline$l_{\mathrm{n}}(\mathrm{cm})$ & 0.54 & 0.85 & 1.25 & 1.50 \\
\hline$N_{\text {l. }}$ (\# periods) & 30 & 23 & 20 & 20 \\
\hline$B_{11}(\mathrm{kG})$ & 2.0 & 2.0 & 2.0 & 2.0 \\
\hline$\lambda_{s}(\mathrm{~cm})$ & 12.9 & 15.5 & 18.7 & 21.1 \\
\hline$f(\mathrm{GHz})$ & 298 & 285 & 288 & 300 \\
\hline$\eta_{r}$ & $4.1 \%$ & $3.0 \%$ & $3.1 \%$ & $3.0 \%$ \\
\hline$\delta \gamma_{2} /\left(\gamma_{-}-1\right)$ (tota $)$ & $\pm 0.8 \%$ & $\pm 0.8 \%$ & $\pm 0.8 \%$ & $\pm 1.0 \%$ \\
\hline beam/wall clearance $(\mathrm{mm})$ & 0.2 & 0.88 & 1.7 & 2.2 \\
\hline$\theta_{\text {jaterept }}(\operatorname{deg})$. & \pm 2.3 & \pm 3.5 & \pm 5.5 & \pm 6.0 \\
\hline$P_{\text {will }}^{\prime \prime}\left(\mathrm{W} / \mathrm{cm}^{2}\right)^{\prime \prime}$ & 177 & 72 & 24 & $<10$ \\
\hline$P_{\text {iin }}(\mathrm{MW})$ & 3.9 & 6.9 & 9.3 & $<8.9$ \\
\hline$P_{\text {int: }}(\mathrm{MW})$ & 0.75 & 0.90 & 1.00 & 1.02 \\
\hline$\eta_{T}(92 \%$ beam energy recovery $)$ & $35 \%$ & $28 \%$ & $28 \%$ & $28 \%$ \\
\hline$\phi_{1,1}(\mathrm{kV})$ & 60.5 & 71.5 & 93.5 & 110 \\
\hline
\end{tabular}

"Injected beam thickness is $1 \mathrm{~mm}$ in all cases.

"Used $\sigma$-values (conductivity) reduced $\times 2$ from textbook values.

The more recent approach is to use higher voltage (stiffer) beams and larger waveguide gaps, as identified in the second, third, and fourth columns of Table I. The feasibility of sheet beam transport for parameters relevant to these three designs is addressed in a subsequent section.

The current density for each design in Table $I$ is well below the limit at which space charge would affect either the FEL interaction efficiency or the equilibrium beam thickness $\left(b_{\text {beam }}\right)$. The estimates of total beam axial energy spread include all contributing effects, including finite beam emittance, space charge depression, and finite beam thickness in the inhomogeneous wiggler fields. Finally, our calculations of the effects of wiggler tapering indicate that stable single-mode equilibria exist with intrinsic efficiencies approximately three times the untapered FEL designs. Preliminary time-dependent simulations, however, have not yet demonstrated how oscillators evolving from noise can access these equilibria. This is a subject for future study. Hence throughout the remainder of this paper we will concentrate our design discussions to untapered FEL oscillators.

\section{Thermal Issues}

As described in [12], we have completed a conservative engineering heat transfer analysis of the proposed FEL configuration to determine what level of thermal heat flux one can confidently remove from the cavity walls. The conclusion to that study was that steady-state fluxes up to $1500 \mathrm{~W} / \mathrm{cm}^{2}$ are well within the capabilities of existing thermal engineering. Fluxes approaching $2000 \mathrm{~W} / \mathrm{cm}^{2}$ might be feasible, but are more challenging. It is notable that these predictions are in good agreement with recent gyrotron cooling experience at Varian, to the extent that the estimated local heat flux where gyrotron cavity failure occurred was found to be approximately $5 \mathrm{~kW} / \mathrm{cm}^{2}$ [2]. Furthermore, the probable failure mode for those cavities was cyclic fatigue rather than steady-state thermal stress.

Having established the capabilities for thermal heat removal, we turned our attention to evaluation of candidate heat sources in our FEL configuration. The sources of heat which we considered included thermal management in the electron gun, in the microwave cavity, and in the wiggler. We consider these components one at a time, followed by the identification of a thermal management question which we have not addressed in detail: cooling of the beam collector.

First, while it is true that all thermionic electron guns (pulsed or $\mathrm{CW}$ ) involve heated cathodes, this is not a new thermal management problem. As discussed in the section on beam generation, our preliminary gun design efforts indicate that the desired sheet beam electron guns will involve emission current densities at the cathode of less than $2 \mathrm{~A} / \mathrm{cm}^{2}$. This level is well within existing thermionic gun experience from the point of view of thermal management.

Secondly, we consider the issue of heat generated by the magnet. Measurements on the University of Maryland's "current sheet" electromagnet wiggler [3], [4] have demonstrated that wiggler fields of $2.0 \mathrm{kG}$ are achievable with approximately $3 \mathrm{kA}$ of wiggler current. From measurements of wiggler resistance, one can conservatively estimate the total heat produced by the wiggler to be less than $10 \mathrm{~kW}$ per wiggler (for a 20-period wiggler), or less than $250 \mathrm{~W}$ per half-period. Present wiggler designs would easily allow for at least three 1-mm-diameter cooling channels per half-period. For 5 -cm-wide wigglers, this corresponds to a cooling surface area of approximately $5 \mathrm{~cm}^{2}$ per half-period. Convective heat removed obeys the relation:

$$
Q(\mathrm{~W})=h A\left(T_{\text {fluid }}-T_{\text {surface }}\right)
$$

where $h$ is the convective film coefficient, $A$ is the cooling surface area, and $T_{\text {lluid }}$ and $T_{\text {surface }}$ are the bulk fluid and cooling channel wall temperatures, respectively. An extremely conservative value for the film coefficient using water coolant would be $3 \mathrm{~W} / \mathrm{cm}^{2} \cdot{ }^{\circ} \mathrm{C}$. For this value of $h$ and $A=5 \mathrm{~cm}^{2}$, the $250-\mathrm{W}$ per wiggler half-period could be dissipated by a mere $20^{\circ} \mathrm{C}$ temperature difference between the fluid and the channel wall. The conclusion to this exercise is that thermal management of the heat generated by the magnet should be a relatively straightforward matter by present technology standards. The argument is even further strengthened by recent research in superconducting and permanent magnet wigglers, indicating that either one of these other technologies may be an acceptable alternative to the room-temperature electromagnet short-period wiggler. In such cases the issue of magnet heat is moot.

Thus we consider the issue of cavity wall heating. There are two possible sources for such heat: 1) RF ohmic losses, and 2) beam current interception (body current). 
For the case of RF wall losses, a quick inspection of Table I reveals that in all four designs the wall losses are less than $200 \mathrm{~W} / \mathrm{cm}^{2}$. These fluxes were conservatively calculated using a wall conductivity value of $2.5 \times 10^{17}$ $\mathrm{s}^{-1}$ (cgs), which is a factor of two smaller than the textbook conductivity of copper [26]. For the higher beam voltage FEL designs, the RF wall heat fluxes are essentially negligible when compared with the $1500 \mathrm{~W} / \mathrm{cm}^{2}$ dissipation limit described in [12]. This would be especially true for the larger gap spacings possible with a superconducting wiggler (see the section on wiggler magnet technology). Finally, it is emphasized that these low-RF thermal heat load estimates have been achieved without invoking the need for corrugated cavity walls and hybrid transverse modes. This is desirable, since theoretical estimates indicate that even shallow wall corrugations are likely to induce backwards wave "orotron" instabilities for such high energy beams [27].

The remaining agent for cavity wall heating is beam interception. Since the short-period wiggler FEL (as with a CARM) requires beam voltages between 0.5 and 1.0 MV and several to tens of amps of (average) beam current, thermal stability of the cavity requires that body currents be less than approximately $2.5-5 \mathrm{~mA} / \mathrm{cm}^{2}$, depending on beam voltage. Our research during the past year has concentrated on determining whether regimes exist in which there is virtually zero body current for wiggler-focused sheet beams. As discussed in the following section of beam transport, we have verified both theoretically and experimentally that such a regime does exist, provided that the beam emittance at injection is small enough. Thus the issue is not whether wiggler focusing can maintain small enough body currents-that capability has been convincingly established by our research. Instead the issue is whether high-voltage sheet beam electron guns can be designed to deliver the requisite beams. We will discuss this latter question in the section on beam generation. The conclusion drawn from our preliminary gun design efforts, however, is that multianode guns using electrostatic focusing should be capable of meeting these needs.

One critical area of challenging thermal management which we have not yet addressed concerns cooling needs in the beam collector (cf. Fig. 1). Due to the proposed use of depressed collection, it is expected that the total deposited beam power-handling capability of the FEL collector will not be significantly greater than that required for comparable gyrotron tubes. As discussed in the section on spent beam energy recovery, however, the exit energy spread in the FEL beam is much smaller than in comparable sources having higher intrinsic interaction efficiencies. This may result in a significantly higher concentration of thermal loading in the FEL collector. Whether this is true, and, if so, whether the collector's thermal management has a practical solution, is a subject for serious future consideration.

Finally, virtually any candidate source of $(\geq) 1-\mathrm{MW}$ $\mathrm{CW}$ (or average) power at ( $\geq$ ) $300 \mathrm{GHz}$ must ultimately address the rather daunting scenario of catastrophic loss of beam focusing fields. Whether such a beam strike will result in catastrophic damage to the tube depends on the speed with which beam voltage power supplies can be shut down and/or crowbarred. Also, it depends on whether the undesired beam strike occurs on a surface which is actively cooled or on a surface where cooling is only by passive conduction.

\section{BEAM Transport}

\section{A. Theory}

The idea of using sheet or ribbon electron beams in high-power vacuum devices is not new. Historically, numerous applications have been suggested and a few investigated. The difficulty of such a configuration, however, arises when the question of beam focusing is addressed. In most previous work, an axial guide field provided the beam focusing (cf. [6, and references cited therein]). This approach was generally abandoned, though, due to beam distortion or disruption via the diocotron (or related) instability. In FEL's, on the other hand, beam focusing can be accomplished with the wiggler fields. In many cases, this wiggler-focusing force is quite substantial and can obviate the need for an axial guide field. One is therefore led to expect that focusing with planar wigglers would be a natural configuration for stable propagation of sheet beams. This hypothesis has been confirmed by our own research [6].

The principle of wiggler focusing has been derived previously [28]. We briefly review selected parts of that derivation to develop the expression forms most convenient to our subsequent experimental data analyses. For a magnetic field produced by an ideal planar wiggler with the form:

$$
\underline{A}_{w}=\frac{B_{w}}{k_{w}} \cosh \left(k_{w} y\right) \cos \left(k_{w} z\right) \hat{e}_{x}, \quad k_{w} \equiv \frac{2 \pi}{l_{w}}
$$

the wiggler-focusing force on an electron can be conservatively approximated as

$$
F_{y} \approx-\left[\frac{e^{2} B_{w}^{2}}{\gamma m c^{2}} \cos ^{2}\left(k_{w} z\right)\right] y .
$$

This expression actually underestimates the confining force for electrons which stray near either magnet (and thus near a waveguide wall). Here we have defined the small transverse dimension (between the upper and lower magnets) as the " $y$ "'-dimension. Using the force expression in (2), the wiggler focusing of electrons (in the narrow transverse dimension) manifests itself in "betatron" orbits which obey the equation [6], [28]:

$$
\frac{d^{2} y}{d t^{2}}=-\omega_{\beta}^{2} y, \quad \omega_{\beta} \equiv \frac{e B_{w}}{\sqrt{2} \gamma m c}
$$

where the motion has been averaged over one wiggler period (hence we are assuming that the betatron period is much longer than the wiggler period). This equation can 
be solved for electrons entering with arbitrary initial location $\left(y_{0}\right)$ and injection angle $\left(\theta_{y 0}\right)$ :

$$
y(z) \approx \mathcal{Y}_{\beta} \sin \left(\frac{2 \pi z}{\lambda_{\beta}}+\psi_{\beta}\right)
$$

where

$$
\begin{aligned}
& \mathcal{Y}_{\beta}=\sqrt{y_{0}^{2}+\left(\frac{\lambda_{\beta} \theta_{w 0}}{2 \pi}\right)^{2}} \\
& \psi_{\beta}=\tan ^{-1}\left(\frac{2 \pi y_{0}}{\lambda_{\beta} \theta_{10}}\right) \\
& \lambda_{\beta}=\frac{2 \pi \sqrt{2} \beta \gamma m c^{2}}{e B_{11}}=\text { betatron period. }
\end{aligned}
$$

Equation (4a) is equivalent to [28, equation (10)]. Here, however, we have explicitly added the solution for the betatron amplitude (4b) for an electron injected with arbitrary values of $y_{0}$ and $\theta_{10}$. Now, assuming we can neglect space-charge effects, there will be no wall interception as long as the betatron amplitude, $\mathcal{Y}_{\beta}$ is less than half of the waveguide gap, $b_{r f} / 2$. Generally, the least confined electron will be an edge (largest $y_{0}$ value) electron. Thus we can solve for the limiting divergence angle at which this least confined electron intercepts the cavity wall. If the beam is relatively monoenergetic (which is the case for high-voltage thermionic guns), one can identify a region in the beam's $y-\theta$ trace space (i.e., electron trajectory's position versus divergence angle) injection for which there is no body current. As a specific example, we have done this for the $850-\mathrm{kV}$ FEL design of Table I. The results are plotted in Fig. 2. Clearly, the FEL design values for injected beam of $\theta_{y 0} \leq \pm 2^{\circ}$ and $2 y_{0}=1 \mathrm{~mm}$ are conservative in divergence angle by a factor of $\sim 3$ and conservative in beam thickness by a factor of $\sim 5$. As described later, the use of a superconducting wiggler can increase both of these safety factors by at least two.

Before discussing our experimental measurements of wiggler-focused sheet beam body current, we consider several other theoretical effects. First, the issue of space charge is discussed. For wiggler-focused sheet beams, space-charge forces are negligible if [6]

$$
\frac{\omega_{p 0}^{2}}{\gamma^{3}} \ll \omega_{\beta}^{2} \text {. }
$$

For quantitative purposes, (5) can be written as

$$
J_{0}\left(\mathrm{~A} / \mathrm{cm}^{2}\right) \ll(232) \gamma \beta B_{w}^{2}
$$

where $B_{11}$, the wiggler field amplitude, is expressed in kG. The "Brillioun"' current density ( $J_{b}=232 \gamma \beta B_{w}^{2}$ ) is plotted in Fig. 3 versus $B_{11}$ for several beam energies between 0.2 and $1.0 \mathrm{MeV}$. Clearly the FEL designs of Table I satisfy (5) or (6) by over an order of magnitude. The conclusion is that space charge forces can be confidently neglected when estimating wiggler focusing effects in the 300-GHz 1-MW FEL's.

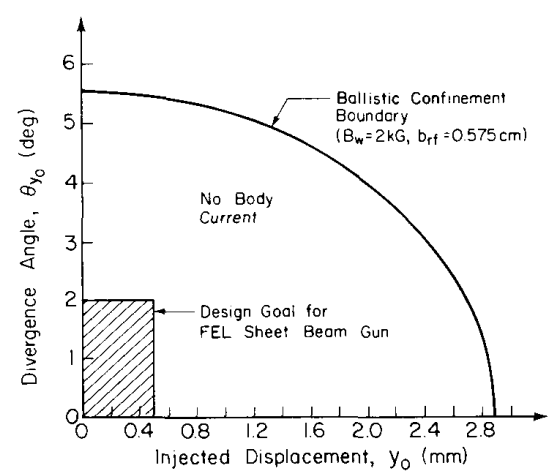

Fig. 2. Injected beam trace space $(y-\theta)$ for which tenuous-beam (ballistic) wiggler-focusing theory predicts no body current. Trace space boundary corresponds to FEL parameters in third column (i.e.. $850 \mathrm{kV}$ ) of Table I. Shaded region represents ultimate design goal for FEL sheet beam thermionic gun.

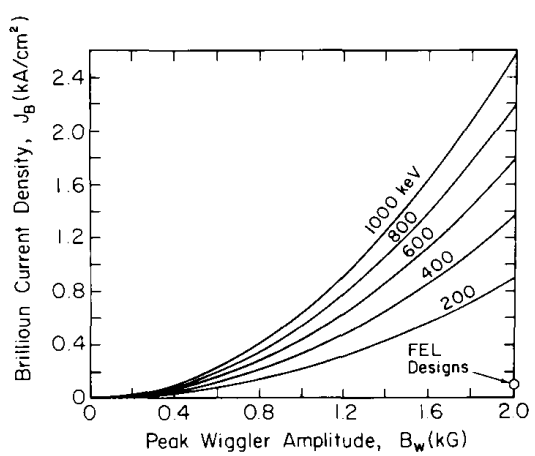

Fig. 3. Theoretical curves, parameterized by beam energy, of "Brillioun" current density, where beam space-charge forces are in equilibrium with wiggler-focusing forces. Open circle represents maximum design current density for FEL sheet beam gun.

There are several other important realistic effects which will add corrections to the wiggler-focusing formulas presented in the preceding. These include radiation (RF) field forces, entrance tapers, wiggler field errors, etc. To theoretically estimate these effects, a nonlinear three-dimensional numerical simulation of beam focusing is being conducted. The code has been described in the literature (cf., for example, [29]) and has been benchmarked against numerous FEL experiments and other codes. At the time of this writing, both RF field and entrance taper effects have been investigated. Since these studies will be described in detail in a subsequent publication, we will just summarize the results here. First, for an injected beam quality which satisfies the unperturbed ballistic confinement conditions (i.e., just wiggler fields, without RFcf. Fig. 2, for example), there is no increase in body current due to the addition of RF fields. This is a direct consequence of the fact that these high-voltage low current density beams are very stiff and the interaction efficiency, $\eta_{e}$, is small. Secondly, with too long of an entrance taper, avoidance of body current imposes severe constraints on beam quality. As one might expect, too long of an en- 


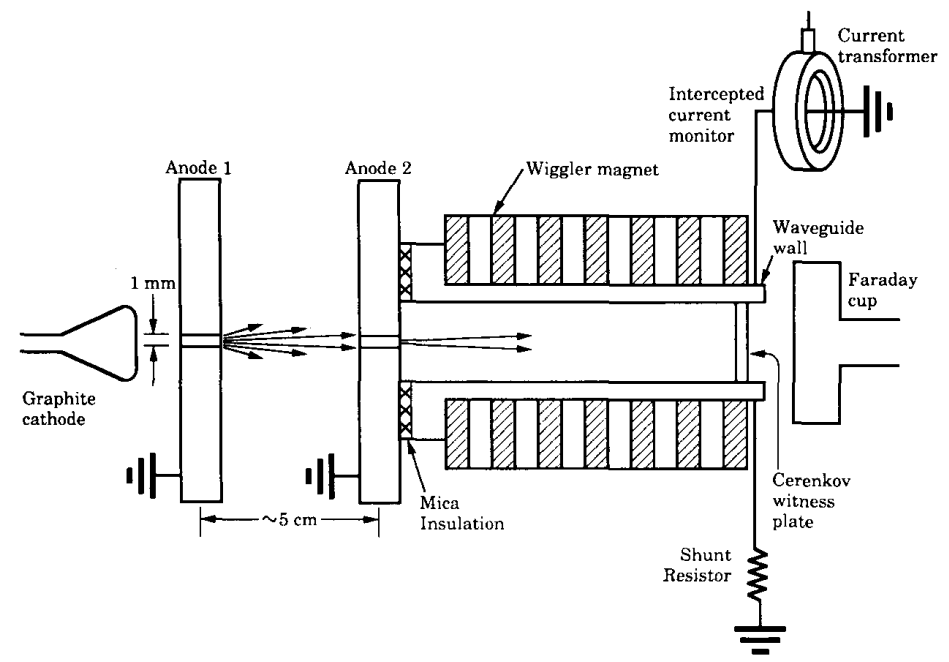

Fig. 4. Experimental configuration for wiggler-focused beam transport experiments. For large emittance beam, anode 2 was removed and wiggler/waveguide assembly was placed adjacent to anode 1 .

trance taper provides greater opportunity for the beam envelope to expand prior to reaching full strength wigglerfocusing. For the beam and wiggler parameters of Table I, however, a short one- or two-half-period taper is adequate for beam entrance matching without affecting beam confinement.

\section{B. Experiments}

Our most recent beam transport experiments have been based on slight modifications of the early configuration of [6]. For these studies, a 50-ns pulse line accelerator and field emission diode generated a short intense beam with a peak voltage of $400-500 \mathrm{kV}$. This estimate of beam voltage was confirmed in several ways, including careful thin foil range/energy measurements. Early experiments [6] used a masking anode with a series of small holes to generate many closely space beamlets. These beamlets merged together shortly after entering the waveguide region to form a sheet beam. Our more recent experiments have replaced the series of holes with a rectangular slit in the anode, thus starting out with a true sheet beam. In addition, we have changed from a five-period wiggler to a ten-period wiggler.

A configuration schematic has been illustrated in Fig. 4. Note that in Fig. 4 we have isolated the waveguide body from the anode and installed a diagnostic to measure the body current. Seven months of intensive effort were spent painstakingly reducing noise signals, eliminating capacitive coupling effects, and improving statistics for measuring small body currents. These body currents were measured for two types of beams: i) A high current density $\left(J \sim 0.5-1.5 \mathrm{kA} / \mathrm{cm}^{2}\right)$, large emittance $\left(\theta_{100} \leqslant\right.$ $\pm 5^{\circ}, \epsilon_{y} \approx 175 \pi \mathrm{mm} \cdot \mathrm{mrad}$ ) beam associated with the single-anode configuration of Fig. 4 (i.e., remove anode $\# 2$ and place wiggler assembly adjacent to anode \#1), and ii) a low current density $\left(J-65-130 \mathrm{~A} / \mathrm{cm}^{2}\right)$, low emit- tance $\left(\theta_{y 0} \leqslant \pm 1^{\circ}, \epsilon_{y} \approx 35 \pi \mathrm{mm} \cdot \mathrm{mrad}\right)$ beam achieved with the double-anode configuration of Fig. 4.

As a result of our efforts at noise reduction, we were finally able to resolve body currents down to a minimum detectable fractional current of

$$
I_{\text {body }} / I_{\text {beam }} \leqslant 0.5 \% \text {. }
$$

The body current data is plotted in Fig. 5. It is evident that the single-anode beam (high-density, large emittance) had appreciable wall interception ( $-20 \%$ of injected current ), even for $2-\mathrm{kG}$ wiggler fields. This is in strong contrast to the double-anode beam (low density, small emittance), which had zero body current (within the $0.5 \%$ measurement resolution) at wiggler fields above 1.8 $\mathrm{kG}$. These data are consistent with theory in several respects. In Fig. 6 we have made a plot similar to Fig. 2, showing the zero-body-current regime for a wiggler-focused $500-\mathrm{kV}$ sheet beam with $B_{w}=2.0 \mathrm{kG}$ and a $3.2-$ $\mathrm{mm}$ waveguide gap (this was the gap used for the transport experiment). In this case, the single-anode beam extends outside the limiting boundary, while the double-anode beam is located well within the interception-free region. It should also be noted that Fig. 6 assumes that space-charge forces can be neglected. Looking at Fig. 7, it is clear that while this is a safe assumption for the double-anode beam, space charge expansion may play an important role in the body current for the single-anode beam, even at wiggler field strengths approaching $2 \mathrm{kG}$. These experiments are discussed further in [30].

At this point, we should compare the experimental parameters to the parameters desired for the point designs of Table I. First, the current density $\left(\sim 65 \mathrm{~A} / \mathrm{cm}^{2}\right)$ and the divergence half-angle $\left(\sim \pm 1^{\circ}\right)$ for the double-anode experiments were comparable to the FEL design specifications ( $~-85-150 \mathrm{~A} / \mathrm{cm}^{2}$ and $<2^{\circ}$, respectively) at beam injection. Similarly, the experimental beam energy 


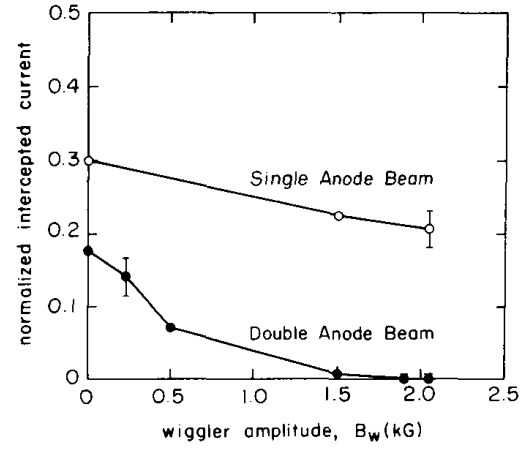

Fig. 5. Fractional body currents for single and double anode beams. Minimum resolution limit for small body currents was $\pm 0.5 \%$ of injected current.

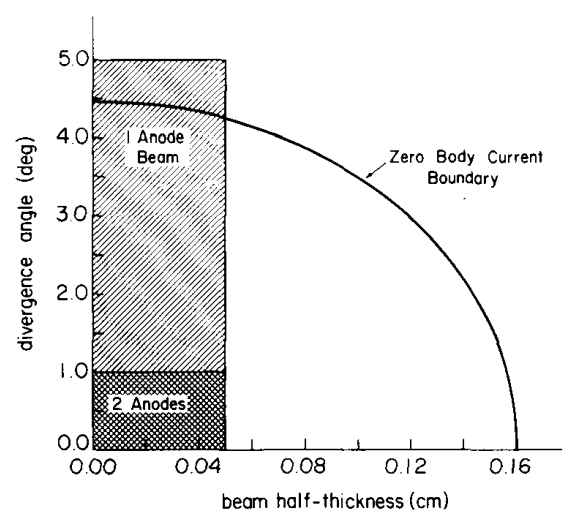

Fig. 6. Zero-body-current trace space for injected beam corresponding to beam transport experiments. Shaded regions represent estimates of single and double anode beam characteristics. Theoretical boundary line neglects space-charge effects.

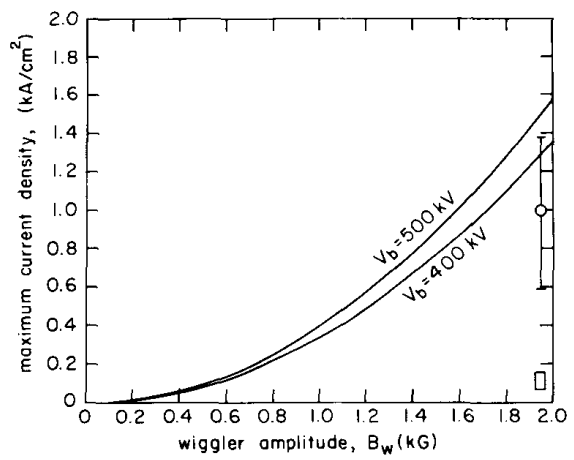

Fig. 7. Comparison of theoretical ("Brillioun") maximum focusable current density to estimates of experimental injected current density. Open circle represents single anode beam, while open rectangle represents double anode beam.

of $\sim 500 \mathrm{kV}$ was comparable to the specifications of Table I. Note that the experimental waveguide gap of 3.2 $\mathrm{mm}$ is smaller than the waveguide gaps specified for FEL designs with beam energies greater than or equal to 600 $\mathrm{kV}$. In this sense, the experiments represent a verification of sheet beam transport under conditions more demanding than one would expect in an optimized ultimate device. It is equally important, however, to emphasize that the 2.2$\mathrm{mm}$ waveguide gap required for the $500-\mathrm{kV}$ design of Table $\mathrm{I}$ is considerably smaller than that used in the experiment. Consequently, the 500-kV design has been judged as probably too optimistic.

So far, we have not addressed the subject of horizontal, or wiggle-plane, focusing. From the point of view of beam interception, there are several reasons why this is not a critical issue. First, for large aspect ratio sheet beams (width $\gg$ thickness), it is straightforward to show that the space charge forces in the horizontal (or " $x$ ") dimension are dramatically reduced by image charges in the upper and lower waveguide walls [5]. Consequently, for our low-current density sheet beams, the imposition of a modest $\sim 100 \mathrm{G}$ " $\mathrm{dc}$ " bias field at the beam edge should be sufficient for horizontal confinement. Schematically, this concept is illustrated in Fig. 8(a) and discussed further in [5].

For permanent-magnet wigglers using low-permeability materials, the addition of this modest edge-focusing field component should be a straightforward matter. For electromagnets (either room temperature or superconducting) using high-permeability inserts, an effective dc field component is achievable [31] by staggering the high$\mu$ inserts, as shown in Fig. 8(b). Measurements of our own wigglers with such staggered inserts confirm the existence of the effective dc edge bias fields. Actual beam transport experiments with such magnets is a subject for future study. Finally, as discussed in the section on cavity design, it is presently expected that the FEL cavity will be an "open-sided" variety where the sidewalls are removed and replaced by small lips in the upper and lower waveguide walls. Hence, for practical purposes, there will not be any sidewalls in the beam's vicinity. Consequently, beam interception on the sidewalls will not be an issue for cavity thermal management.

In conclusion, our completed theoretical and experimental research convincingly argues that body currents for wiggler-focused sheet beams can be kept to virtually zero, provided a sufficient quality beam can be generated. As we will discuss in the next section, our preliminary gun design efforts indicate that the required $1.0-\mathrm{mm}$-thick 0.5-1.0 MV sheet beams with divergence angles $\theta_{10} \leqslant$ $\pm 2^{\circ}-3^{\circ}$ are feasible using multianode electrostatic focusing. For completeness, we are conducting numerical studies of beam transport, including entrance tapers, field errors, and other realistic effects. Since our FEL designs call for quick entrance tapers $\left(\frac{1}{2}-1\right.$ period $)$ and short wigglers ( -20 periods), however, there is no reason to expect the aforementioned conclusions to fundamentally change. Finally, even more compelling is the fact that with superconducting wigglers, a larger waveguide gap is possible for the same wiggler field strength and wiggler period (cf. Fig. 2). In fact, for a superconducting wiggler, the most important constraint on beam emittance involves maximizing FEL efficiency by minimizing axial energy 


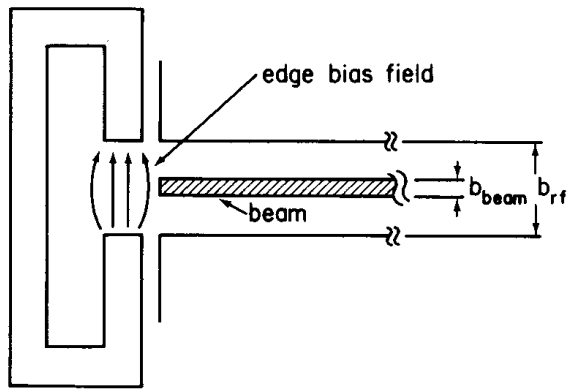

(a)

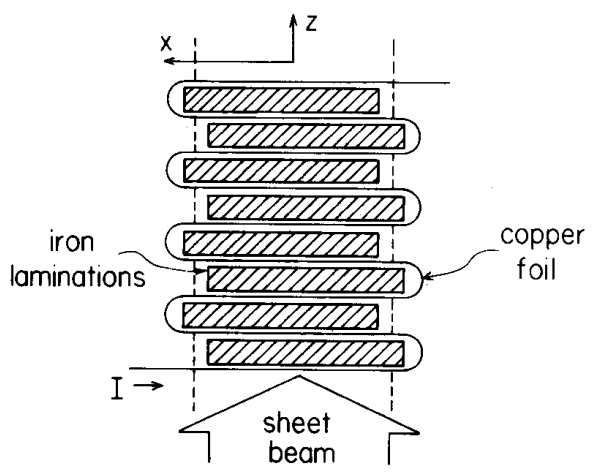

(b)

Fig. 8. Wiggle-plane (horizontal) sheet beam-focusing techniques. (a) Edge "bias" field. (b) Offset pole pieces.

spread due to finite beam thickness in the homogeneous $B_{w}(y)$. The body current and RF wall losses for such a large gap become secondary, almost irrelevant, concerns. We will explore this subject in a subsequent section, as well as in a later publication.

\section{High-Voltage CW Sheet Beam Generation}

As discussed in the previous sections on conceptual design and beam transport, our FEL's will require a $\mathrm{CW}$ thermionic Pierce gun with a beam thickness $b_{\text {beam }} \sim 1$ $\mathrm{mm}$, beam voltage $\sim 0.5-1.0 \mathrm{MV}$ (tunable), linear current density $i \sim 7.5-15.0 \mathrm{~A} / \mathrm{cm}$ (area current density $J$ $\sim 75-150 \mathrm{~A} / \mathrm{cm}^{2}$ ), and a maximum divergence angle $\sim \pm 2^{\circ}$. Present state-of-the-art capabilities [32] set the acceptance limit on maximum electric field for $\mathrm{CW}$ operation at $\sim 100 \mathrm{kV} / \mathrm{cm}$. Finally, to realize the maximum achievable FEL intrinsic (or electronic) efficiency, we require the axial energy spread of the beam in the interaction region to be $\Delta \gamma_{\Sigma} /\left(\gamma_{z}-1\right) \leq \pm 2 \%$ [11].

To achieve these characteristics, it was suggested that we consider a multianode Pierce gun with electrostatic focusing [32]. The theoretical formalisms to design such structures have been known for some time [33]-[40]. Furthermore, the accuracy of these design techniques has been experimentally verified in relativistic [36], [39], [40] as well as many nonrelativistic guns. The basic principle for generating a compressed $\mathrm{CW}$ high-voltage electron beam which is tunable in voltage (i.e., the voltage can be changed at constant current) is to generate an electrostatically compressed beam at low to modest voltage (while the beam is still soft) and then pass it through a series of electrostatic lenses that accelerate the beam while maintaining its focus. In this process, the total beam voltage becomes spread out over a larger distance than for a single-anode gun, thus keeping electric fields to acceptable levels. In addition, the high beam velocity means that the space-charge perturbation on the vacuum fields is negligible in the gap between the penultimate and final anodes. Hence the potential difference between the last two anodes can usually be varied over a modest range without significantly affecting the beam current or the beam emittance.

We have taken a cursory look at the feasibility of such high-voltage sheet beam gun design using a well-tested electron optics code [41]. An example of a 1.0-MeV sheet beam gun design is illustrated in Fig. 9. The emittance for this beam was $13 \pi \mathrm{mm} \cdot \mathrm{mrad}$. The remaining beam characteristics at the gun exit are tabulated in Table II alongside the desired specifications presented earlier. As one can clearly see, almost all the desired specifications have been met or exceeded by this first design attempt. In view of the brevity of time spent to achieve this design, it is expected that the $50 \%$ increase in current density and the one degree decrease in divergence angle would be readily achieved in a more systematic, extended study.

We now consider several additional issues for the gun design. First, the solution of Fig. 9 was tested for sensitivity to small changes in electrode position, to numerical convergence criteria, and to the computation of self-magnetic forces. In all cases, the solution was found to be stable and robust. It is also emphasized that previous studies [42] have established the accuracy of the code for designing similar high-voltage (i.e., $-500 \mathrm{kV}$ ) thermionic electron guns.

Secondly, substantive differences exist between the sort of gun proposed in Fig. 9 and any previous microwave tube gun. These differences include the very high voltage (which yields a stiff beam with small angular divergence spread), the large clearances between beam edges and anode apertures (keeping aberrations small), and the low current density (keeping space charge negligible). Thus certain limitations on achievable beam quality based on previous gun manufacture will not be applicable here.

Thirdly, we have considered possible sources of transverse momentum spread and "halo" current. These have included finite cathode temperature, cathode surface roughness [43], and transverse field effects at the edges of space-charge-limited emitting strips. First, we can expect finite cathode temperature to yield transverse energy characterized by the cathode temperature:

$$
T_{1}^{\text {cathode }} \approx 0.1 \mathrm{eV} \text {. }
$$

From [43] we can estimate cathode surface roughness to contribute to finite transverse energy of order:

$$
T_{\perp}^{\text {surface }} \approx 3 \mathrm{eV}
$$




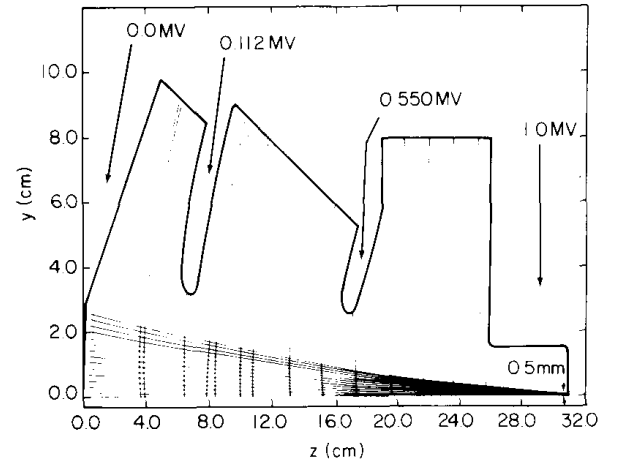

Fig. 9. Simulation solution for multi-anode high-voltage sheet beam gun. Nonthermal version shown used 14 rays to simulate beam. Solution was identical to that based on 100 rays. Emittance was $13 \pi \mathrm{mm} \cdot \mathrm{mrad}$.

TABLE II

SHEFt BeAM GINCHARACTERISTICS

\begin{tabular}{lcc}
\hline \multicolumn{1}{c}{ Quantity } & & $\begin{array}{c}\text { Ist-cut Design } \\
\text { Value }\end{array}$ \\
\hline Thickness & $1.0 \mathrm{~mm}$ & $1.0 \mathrm{~mm}$ \\
Beam voltage & $0.5-1.0 \mathrm{MV}$ & $0.7-1.0 \mathrm{MV}$ \\
Voltage tuning & $\geq 100 \mathrm{kV}$ & $300 \mathrm{kV}$ \\
Linear current density & $7.5-15 \mathrm{~A} / \mathrm{cm}$ & $5.0 \mathrm{~A} / \mathrm{cm}$ \\
Divergence angle & $\leq \pm 2^{\circ}$ & $\leq \pm 3^{\circ}$ \\
Maximum electric field & $\leq 100 \mathrm{kV} / \mathrm{cm}$ & $\leq 70 \mathrm{kV} / \mathrm{cm}^{-1}$ \\
Beam energy spread & $=0$ & $<0.05 \%$ \\
Axial energy spread in FEL & $< \pm 2 \%$ & $\pm(1.2-1.9) \%$ \\
Cathode emission current density & $<10 \mathrm{~A} / \mathrm{cm}^{2}$ & $-1 \mathrm{~A} / \mathrm{cm}^{2}$ \\
\hline
\end{tabular}

Here it has been assumed that any surface roughness can be characterized by "feature" scale size $l_{s}$, where $l_{s} \leq$ 0.001 in $(0.025 \mathrm{~mm})$. Finally, we note that electrons at the beam edge see a defocusing potential depression of the vacuum field. We have conservatively included this factor in our estimates of body current by assigning this entire potential drop to the transverse energy of edge electrons. Thus for the solution shown in Fig. 9, this yields an upper bound on the effective transverse "temperature" for edge electrons of

$$
T_{\perp}^{\text {edge }} \approx 300 \mathrm{eV} .
$$

To see how these thermal models affect body current, we repeated the simulation of the gun in Fig. 9, using 100 nonthermal rays. Then each ray was assigned a Gaussian spread in divergence angle, characterized by the standard deviation angle corresponding to $T_{\perp} \approx 3 \mathrm{eV}$ for the bulk of electrons, and $T_{\perp} \approx 300 \mathrm{eV}$ for electron beamlets close to the emitting strip edge. The individual gaussians are then summed to form a composite angular divergence distribution as shown in Fig. 10. When one calculates the amount of halo current in the "wings" of this composite distribution which can strike the wall, the resulting body current is less than $10^{-7}$ of the injected beam current. Since such a small body current ( $\lesssim 1 \mu \mathrm{A}$ ) will be practically immeasurable, this answer is essentially equivalent to zero body current. (Note: The particle simulations de-

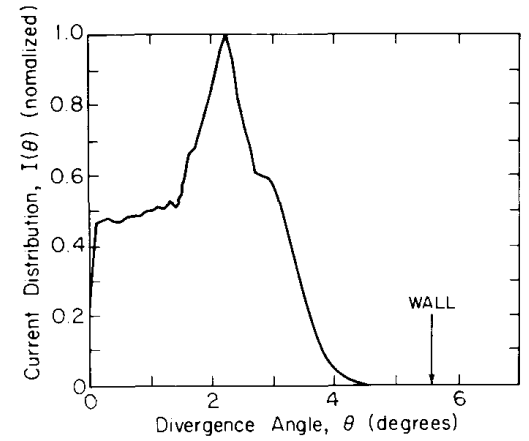

Fig. 10. Injected beam 's divergence-angle current distribution based on 100-ray 850-kV thermal model of gun in Fig. 9. For this gun and 850kV FEL design of Table $I$, only beam current with injected pitch angles exceeding $\pm 5.5^{\circ}$ will intercept cavity body.

scribed in Section IV-A have established that this body current will be uniformly distributed over a one-periodlong section of guide wall near the cavity entrance. Thus body current densities are also well below the $\sim 1$ $\mathrm{mA} / \mathrm{cm}^{2}$ limit.)

As a final check on thermal effects, we have run one additional code simulation for the gun of Fig. 9. This simulation partitions the rays originating at the cathode to simulate thermal effects. The algorithm has been described elsewhere [44]. For this case, a three-way current-ray split [44] was used, with $T_{\perp}=3 \mathrm{eV}$ to conservatively simulate cathode surface roughness effects. Note that such a simulation will self-consistently compute the effects of potential depression at the beam edge. The resulting $y-\theta$ loci of each ray are plotted in the trace space of Fig. 11. Also shown are boundaries for negligible body current conditions based on confining the betatron amplitudes of (4b) inside the waveguide gap without interception. Note that when weighted by the current in each ray, the resultant beam associated with Fig. 11 still has an rms beam thickness of $1 \mathrm{~mm}$. The emittance for this beam was $49 \pi \mathrm{mm} \cdot \mathrm{mrad}$.

\section{Spent-Beam Energy Recovery}

From a study of Table I, it is clear that the short-period wiggler FEL (untapered version) only becomes attractive as a CW radiation source if the spent-electron-beam's energy can be recovered with an efficiency in excess of $80 \%$. This enhances the overall (total) device efficiency to values between 20 and $40 \%$, and reduces the voltage on the main power supply to approximately $100-200 \mathrm{kV}$. The use of multistage depressed collectors for spent-beam energy recovery in low-voltage TWT's is a well-known technique, dating back to the early 1950's [45]. As described in the review article by Kosmahl [46], streaming beams with small axial energy spreads are ideally suited to highly efficient energy recovery. In fact, both theory and experiments have long since established that for lowvoltage streaming beams with exit axial energy spreads $\leq 5 \%$, energy recovery efficiencies in excess of $95 \%$ are achievable [45], [46]. 


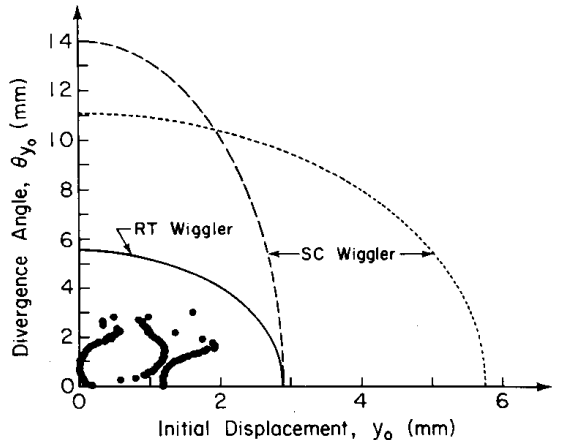

Fig. 11. Zero-body-current trace space boundaries compared with injected $850-\mathrm{kV}$ beam from tetrode gun of Fig. 9. Solid boundary is for room temperature wiggler (RTW) case of Fig. 2. Dotted line is for superconducting wiggler ( $\mathrm{SCW}$ ) with fields equal to $\mathrm{RTW}(2 \mathrm{kG})$ but twice gap spacing. Dashed boundary is for SCW with $l_{w}=1.25 \mathrm{~cm}, b_{, y}=0.575$ $\mathrm{cm}$. and $B_{\mathrm{n}}=6 \mathrm{kG}$. Data points are ray loci for three-ray thermal model $(3-\mathrm{eV})$ simulation of Fig. 9. When weighted by current in each ray. "rms" beam thickness is still approximately $1 \mathrm{~mm}$. Emittance for this beam was $49 \pi \mathrm{mm} \cdot \mathrm{mrad}$

Since the design of depressed collectors is based upon the solution of Poisson's equation, there is no reason to expect a priori that (aside from the issue of secondary electrons) efficient collector design for high-voltage beams is not possible as well. In fact, published claims of such capability have been previously reported [8], [9], [47]. These measurements were done with 2-6 MV, 1-3 A beams which were routed through an extended circuit of many beamline bends and other sources of beam quality depletion. Nevertheless, collector efficiencies of 99.0$99.9 \%$ have been claimed in the absence of lasing, while efficiencies of $95-97 \%$ have been reported during FEL operation. Thus it is expected that the desired $80-90 \%$ collector efficiencies would be achievable in the short-period wiggler FEL, provided that the exit energy spectrum is sufficiently simple (i.e., small energy spreads). This confidence is strengthened by the fact that at most four, and possibly no beam bends are anticipated for the proposed sheet beam FEL.

Thus we turn to the question of the spent-beam energy spectrum for the short-period wiggler FEL oscillator. This is critical to the energy-sorting task, which impacts both the expected achievable collector efficiency as well as the size and complexity of the depressed collector and its power supply. In this regard, one of the many interesting observations to arise from our most recent FEL oscillator simulations is the predicted spent-beam energy distribution. In particular, the 3-4\% electronic efficiencies of the untapered designs in Table I result from downshifting approximately half of the beam electrons by $6-8 \%$ in energy. The remaining electrons exit the device at the original injection energy. An example of this spent beam energy distribution is shown in Fig. 12. Note that the two energy peaks are separated by approximately $6 \%$ in energy, with each peak having an extremely narrow (local) energy spread of less than $1 \%$. This particular example was run for a monoenergetic beam at injection. For beams

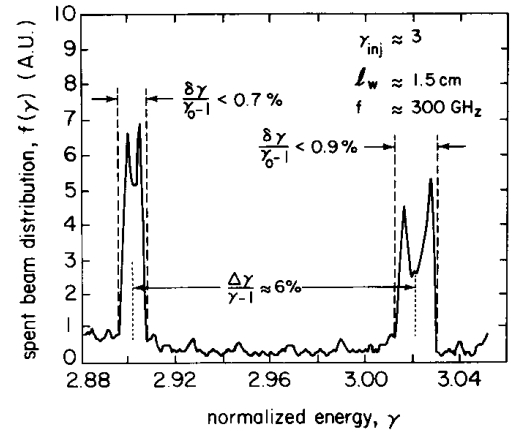

Fig. 12. Typical spent-beam energy distribution for $\eta_{e} \approx 3-4 \%$ in FEL oscillator. Injected beam for this case was cold. with interaction parameters corresponding to fourth column of Table $I$.

with finite energy spread at injection, the result is essentially the same, with each of the two peaks individually having the energy spread of the input beam.

Finally, we consider a recent simulation of spent-beam energy recovery of high-voltage $(\sim 500 \mathrm{kV})$ gyrotron beams [48]. In that work it was established that energy recovery efficiencies of $\eta_{\text {coll }} \geq 80 \%$ should be readily achievable for high-voltage gyrotron beams with practical two-electrode depressed collectors. Furthermore, these simulations were for a spent beam with approximately $\pm 50 \%$ spread in energies. The results of this study add further credence to the expectation that the desired FEL beam collection efficiencies will be achievable with practical depressed collector designs.

In contrast to the optimistic anticipation for high collection efficiencies, thermal management in the collector structure is a topic for serious concern. While the total power in the FEL's decelerated spent beam is comparable to that in high-power millimeter-wave gyrotrons, the FEL's smaller exit energy spread may lead to greater localization of beam power deposition on the collector. This important question, at present, remains unanswered. Future two- and three-dimensional beam simulations are planned to address this issue.

\section{Power Supply Technology}

For both CW FEL's and CARM's operating at voltages $0.5-1.0 \mathrm{MV}$, the fundamental issues for power supply technology are cost and practicality. This is why the subject of spent beam energy recovery is so important. The driving element here is that insulation techniques, power supply size, and cost make a "quantum transition" at voltages exceeding $250-300 \mathrm{kV}$ [49]. In fact, the present state-of-the-art achievement for a commercial dc highvoltage high-power supply is a $700-\mathrm{kW} 700-\mathrm{kV}$ unit consisting of two tanks which fill a $1200 \mathrm{ft}^{3}$ (approximately $36 \mathrm{~m}^{3}$ ) space [49]. Currently, research and development efforts are underway to develop more compact (and less expensive) sources delivering up to $10 \mathrm{MW} \mathrm{dc}$ at $1 \mathrm{MV}$ [49]. Until this development is successful, however, any needs for dc power exceeding $1 \mathrm{MW}$ at voltages above 
$-300 \mathrm{kV}$ would require an outdoor substation. This would be expensive and probably impractical for the intended application. On the other hand, dc supplies near $100-200 \mathrm{kV}$ at power up to $5 \mathrm{MW}$ and/or supplies up to $1 \mathrm{MV}$ at powers $\leqslant 300 \mathrm{~kW}$ are commercially available in a practical, affordable form. Admittedly, the "low-voltage" power supply $\left(\phi_{L V}\right)$ of Fig. 1 is floating at voltages up to $1 \mathrm{MV}$. Consequently, the input power for that floating supply must transmitted through a high voltage isolation $(\sim 1 \mathrm{MV})$ transformer. Nevertheless, the cost, challenge, and efficiency of transforming this ac input power (probably in $\mathrm{SF}_{6}$ insulation) is considerably more straightforward than the task of rectifying many megawatts of power at $1 \mathrm{MV}$ dc [49]. Other options might include the use of a very high frequency switching supply since the isolation task would be made easier at higher frequencies. The cost or availability of this technology has not been extensively investigated. A further possibility would be to employ the electrical circuit of [10], wherein the waveguide body is floated at high positive potential. This latter choice, however, is probably incompatible with the desire for active water cooling of the cavity.

We recall that for a simple depressed collector, the relationship between the low voltage $\left(\phi_{L V}\right)$ and high bias voltage $\left(\phi_{H V}\right)$ of Fig. 1 can be expressed as

$$
\phi_{L V} \approx\left[1-\left(\eta_{\text {coll }}-\eta_{c}\right)\right] \phi_{H V}
$$

where $\eta_{\text {coll }}$ is the collector efficiency and $\eta_{e}$ is the intrinsic or electronic efficiency of the oscillator. Hence from the previous discussion, if $\phi_{H V}$ is between 700 and $1000 \mathrm{kV}$, it is clearly imperative (from a power supply cost and practicality standpoint) to get the quantity $\left(\eta_{\text {coll }}-\eta_{e}\right)$ between 0.7 and 0.8 , respectively (or higher). This need favors highly efficient beam recovery $(\geq 80 \%)$ and low to modest electronic efficiency ( $\leq 10 \%$ ).

To summarize, the question of high-voltage dc power supply technology is primarily one of practicality and cost. Because of the aforementioned facts, it is important with high-voltage ECRH sources ( $\geq 500 \mathrm{kV}$ ) to achieve high spent-beam collection efficiencies ( $\geq 80 \%$ ). For the untapered FEL designs of Table I, we have listed a design goal on collector efficiency of $90-92 \%$. This is $10 \%$ higher than that required to achieve the less expensive power supplies $\left(\phi_{L V}<200 \mathrm{kV}\right)$. It would allow, in fact, the specified FEL designs to utilize even lower voltage supplies with voltages $\sim 100 \mathrm{kV}$. As already mentioned, relaxation of the collection efficiency specification to $80 \%$ would still provide for the cheaper power supply technology. The expected feasibility of achieving the high collector efficiencies has already been discussed in the previous section.

\section{Wiggler Magnet Technology}

Because of the relatively weak wiggler parameter specified for our FEL designs (i.e., $a_{w} \sim 0.1-0.2$ ), our wiggler requirements are relatively modest compared to demonstrated wiggler technologies [50]. Based on our theoretical calculations, our wiggler requirements include $l_{w} \approx 0.5-1.5 \mathrm{~cm}, B_{w} \approx 1.5-2.0 \mathrm{kG}$, and $\delta B_{w} / B_{w}<$ $10 \%$. This latter modest constraint on wiggler field errors is possibly because of the relatively weak wiggler parameter $\left(a_{w} \sim 0.1-0.2\right)$ associated with the choice of the low-gain low-efficiency FEL oscillator. For example, in the designs of Table I, the total values of $\delta \gamma_{z} /\left(\gamma_{z}-1\right)$ $\leq \pm 1 \%$ are primarily dominated by the inhomogeneous wiggler fields sampled by beams with the corresponding equilibrium beam thicknesses given by $b_{\text {beam }}$ (Table I). To have wiggler field errors contribute a negligible addition to this effective beam energy spread requires $\delta B / B \leqslant$ $10 \%$. Note that the stiff beams, short oscillator cavity lengths $\left(N_{w} \leq 20\right)$, and small values of $a_{w}$ ensure that diffusive beam emittance growth due to wiggler field errors will not be significant. Finally, as previously discussed, acceptable beam-matching at the wiggler entrance requires $B_{w}$ (first half-period) $\approx 1 / 2 \times B_{w}$ (bulk); i.e., a short 1/2-to-1-period taper. While this results in a small horizontal beam displacement $(\Delta x-1 \mathrm{~mm})$, the effect is negligible compared to the overall beam and waveguide widths ( $a_{\text {beam }}$ and $a_{r f}$, respectively).

As discussed in [5], we have already demonstrated electromagnet wiggler performance which exceeds these criteria. It is emphasized that this performance has been achieved with fabrication techniques that are rather rudimentary compared to what is available in commercial industry (consider, for example, some of the more sophisticated fabrication techniques used in [50]). Thus improvements well beyond our required specifications will undoubtedly be observed as we improve our fabrication methods.

Other exciting options have been raised by recent advances in small period superconducting (SC) wigglers [51]-[53]. These alternatives are a consequence of the increased field strengths realized with $\mathrm{SC}$ wigglers in practical configurations for periods as short as $0.8 \mathrm{~cm}$. The simplest option would maintain the same FEL parameters as in Table I, except for widening the waveguide gap by a factor of two. For this case, as shown by the dotted boundary in Fig. 11, the zero-body-current trace-space region increases by roughly a factor of two both in divergence angle and beam thickness. The next alternative is to keep approximately the same gap spacings, but increase the on-axis peak wiggler field. Recent work at Brookhaven National Laboratory, for example, is leading to the demonstration of a $0.88-\mathrm{cm}$-period SC wiggler with a $0.48-\mathrm{cm}$ waveguide gap and an on-axis field of $5 \mathrm{kG}$ [53]. From beam confinement considerations, the enhanced wiggler field leads to a significant reduction in body current risks, as shown by the dashed boundary in Fig. 11. The implications for the FEL interaction design are more complex, however, although generally in an advantageous manner. For example, with the higher magnetic fields, one could choose to stay with the low-efficiency $\left(\eta_{e} \leqslant 10 \%\right.$ ) FEL oscillator (this would require a redesign of the interaction length, cavity transmission, etc.), employ high-efficiency beam energy recovery, and 
still keep the high-power dc supply voltage under $200 \mathrm{kV}$. A different approach would be to use the enhanced fields to access the high-gain (Compton) regime. With tapering, it has been demonstrated that single-pass efficiencies of $30-40 \%$ are possible in the regime [23].

This regime of operation, however, is outside the scope of this article. Furthermore, for such efficient devices, beam energy recovery will probably not be able to reduce the voltage on the main power supply (cf. Fig. 1) below $300-400 \mathrm{kV}$. Thus practical implementation of a CW FEL with $\eta_{e} \approx 30-40 \%$ will require additional technological advances in high-voltage high-power dc supplies. These issues and other implications of SC short-period wigglers for sheet beam FEL design will be explored more fully in a future publication.

In conclusion, our requirements on wiggler performance are modest compared to the demonstrated capabilities of several available technologies. Hence wiggler technology is not considered a risk issue for our FEL concept.

\section{Cavity Design}

To pose the problem, what is desired is a cavity with frequency selective feedback (to allow for mode discrimination) as well as the capability to conveniently separate the radiation and electron beam. Our proposed solution is based on some variation of Fig. 13, which we refer to as an "open-sidewall" rectangular cavity.

Reference [16] discusses the fact that in the closed sidewall configuration, numerous transverse modes (for the same axial mode) have comparable linear gain. While the question of whether nonlinear mode suppression would result in a single saturated transverse mode is still unanswered, [16] established that a probable solution would be to replace the sidewalls with small "lips" in the top and bottom cavity walls (as shown in Fig. 13). These lips confine the desired $\mathrm{TE}_{01}$ rectangular mode but diffractively suppress all the unwanted higher-order modes. Fig. 14(a) and (b) conveys this information in terms of the linear gain for the various transverse modes, both with and without sidewalls.

The other advantage of the open-sidewall configuration is that it allows for separating the beam and radiation by either bending the beam or bending the RF in the wiggle plane. Thus, for example, bending the beam would entail extracting the beam from the interaction region through one of the "open"' sidewalls. Again, the $\mathrm{TE}_{01}$ mode would be confined by the "lips" in the upper and lower cavity walls. Once separated, the beam can be transported under wiggler focusing (using a wiggler with longer period to suppress FEL interaction) to the depressed collector. The RF cavity could then be terminated with a quasi-optical mirror arrangement similar to the quasi-optical (QO) gyrotron. This would permit high reflectivity at the desired high-frequency FEL interaction, while diffractively suppressing feedback at the lower frequency mode. Finally, because of the rectangular geometry, it would be convenient to couple out the $-10 \%$ of cavity power through a

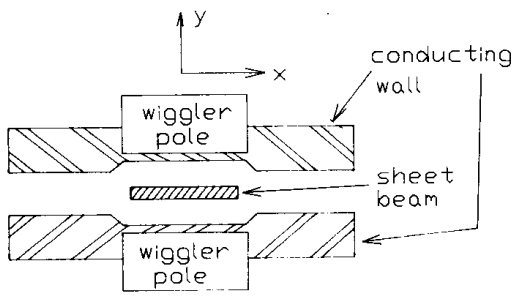

Fig. 13. Open-sidewall rectangular cavity cross section for sheet beam FEL oscillator.

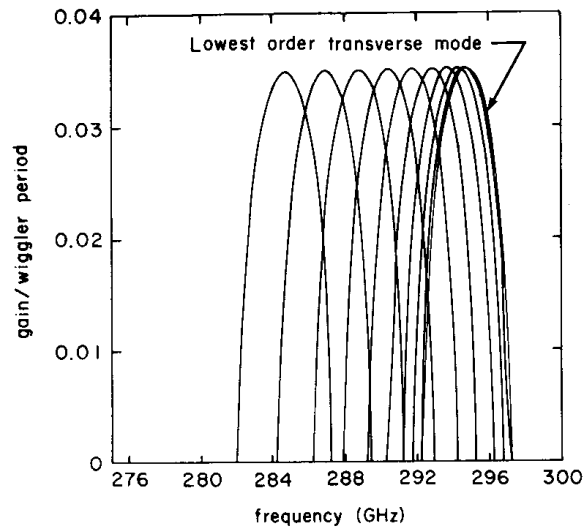

(a)

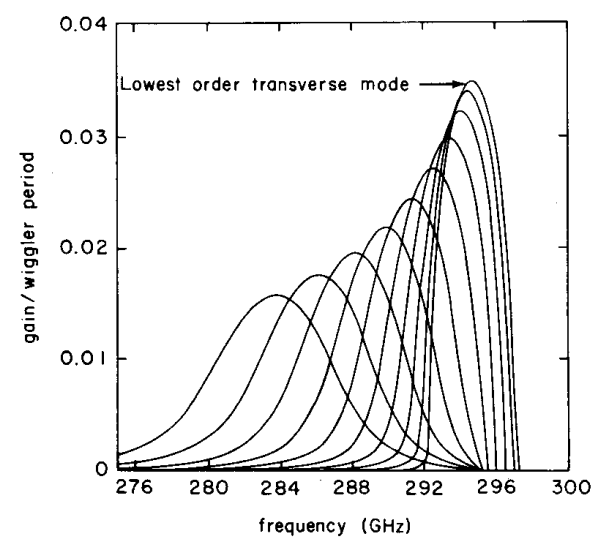

(b)

Fig. 14. Mode control in open-sided rectangular cavity. Comparison of linear gain in transverse modes for rectangular cavity. (a) With closed sidewalls. (b) With open sidewalls.

sidewall, as well. This would avoid the problem of mode purity encountered with present QO gyrotron experiments wherein output power is coupled from the cavity via mirror "spillover."

To summarize, the issue of cavity design feasibility will require careful future study. An "open sidewall" configuration has been identified which satisfies the desire for transverse mode control while providing a means to conveniently separate the beam and the RF. Once separated, the beam can be transported to a depressed collector, while the radiation cavity is terminated in a simple, frequency 
selective, quasi-optical mirror. Radiation power extraction would probably be done via sidewall coupling. Detailed designs for the actual methods of beam and/or radiation "bending," however, represent a very challenging outstanding task. For example, at high frequencies, "bending" the RF wave with the cavity sidelips will have to be done very slowly to avoid mode conversion. Alternatively, while achromatic magnetic lenses have been developed for bending round beams [54], it is not clear how well the concepts transfer to sheet electron beams.

\section{Summary}

To summarize, we have addressed the dominant risk issues for design, fabrication, and operation of near-millimeter megawatt CW FEL oscillators using untapered short-period planar wigglers and sheet electron beams. Ohmic RF cavity losses are conservatively estimated to be extremely low, having an order of magnitude safety factor below practical cooling limits. Theory predicts that sufficiently low-beam emittance at injection will ensure acceptably low (i.e., negligible) body current for wigglerfocused sheet beams. This prediction is strongly supported by experimental measurements on beams with parameters relevant to the proposed FEL designs.

Preliminary studies of high-voltage multianode beam gun design indicate that it will be both possible and practical to generate the required quality $\mathrm{CW}$ beams associated with negligible body currents. These gun design studies have included consideration of thermal beam effects.

For low intrinsic efficiency oscillators, efficient spentbeam energy collection ( $\eta_{\mathrm{coll}} \approx 80-90$ ) will be necessary for enhancement of total system efficiency to practical levels $\left(\eta_{T} \approx 30-40 \%\right)$. Although there may be concern with placing so much relevance on the beam recovery process, this configuration yields a desirable situation in which most of the high-voltage ( $\left.V_{b} \simeq 0.5-1.0 \mathrm{MV}\right)$ beam power is produced by lower voltage $(\sim 100-200 \mathrm{kV})$, practical dc supplies. While more work is required on this issue, the existing experimental and simulation database indicates that the desired collection efficiencies should be achievable with practical collector designs.

The required specifications on wiggler performance are well within the demonstrated capabilities of several available room-temperature magnet technologies. Furthermore, recent advances in superconducting wiggler development portend exciting possibilities for FEL design improvements. The simplest improvements involve larger waveguide gap and greater beam clearance. More substantive FEL revisions involve higher on-axis wiggler fields, higher gains, and/or higher efficiencies. In particular, a strong-pump (Compton), high-efficiency ( $\sim 30 \%$ ) tapered FEL amplifier might be possible using a $\mathrm{CW}$, superconducting, short-period wiggler. These options will be explored in a future publication.

An open-sided resonator cavity concept has been identified. This cavity provides selective mode discrimina- tion, as well as a method for separating the radiation and the electron beam.

Finally, the remaining risk areas yet to be addressed include separation of the electron beam from the output radiation (i.e., either bending the beam or the RF), design of the depressed collector with particular focus on thermal management of the beam dump, transporting the beam from the cavity to the collector, verifying the feasibility of side-focusing with sheet electron beams, and response to a catastrophic loss of beam-focusing fields. This latter question is probably ubiquitous to all candidate sources of very high average-power millimeter waves.

\section{ACKNOWLEDGMENT}

The work described in this article resulted from the suggestions and criticisms of many generous individuals. A partial listing of those to whom the authors are indebted include Drs. W. Lawson, A. Singh, R. Jackson, W. Herrmannsfeldt, and C. R. Chang. The assistance of J. Rodgers, D. Bensen, and J. Pyle is gratefully acknowledged. The careful reading by anonymous reviewers is also graciously appreciated.

\section{REFERENCES}

II] T. Marshall. J. Lebacqz. and T. Godlove. "Electron cyclotron heating-Technology review. " Dept. of Energy Rep.. DOE/ER-0366. pp. 1-25. Apr. 1988.

12] H. Jory. Varian, private communications. 1989

[3] V. L. Granatstein, W. W. Destler, and I. D. Mayergoyz, "'Smal]period electromagnet wigglers for free-electron lasers," Appl. Phys. Lett., vol. 47, no. 6, pp. 643-645. Sept. 15, 1985.

[4] W. W. Destler, V. L. Granatstein, I. D. Mayergoyz, and Z. Segalov. "Near-millimeter free electron laser designs based on measured characteristics of small-period electromagnet wigglers," J. Appl. Phys., vol. 60, no. 2. pp. 521-528, July $15,1986$.

[5] V. L. Granatstein. T. M. Antonsen, Jr., J. H. Booske, W. W. Destler, P. E. Latham, B. Levush, I. D. Mayergoyz, D. J. Radack. Z. Segalov, and A. Serbeto. "Near-millimeter free electron lasers with small period wigglers and sheet electron beams." Nucl. Instrum. Methods, vol. A272, nos. 1/2, pp. 110-116, Oct. 1988.

[6] J. H. Booske, W. W. Destler, Z. Segalov, D. J. Radack, E. T. Rosenbury, J. Rodgers, T. M. Antonsen Jr., V. L. Granatstein, and I. D. Mayergoyz, "Propagation of wiggler focused relativistic sheet electron beams," J. Appl. Phys., vol. 64, no. 1, pp. 6-11, July 1, 1988.

[7] L. R. Elias and G. J. Ramian, "Status report of the UCSB FEL experimental program." in Free Electron Generators of Coherent Radiation, C. A. Brau, S. F. Jacobs, and M. O. Scully. Eds, SPIE vol. 453. Bellingham. WA: SPIE, pp. 137-150, 1983.

[8] L. R. Elias, J. Hu, and G. Ramian, "The UCSB electrostatic acce]erator free electron laser: First operation," Nucl. Instrum. Methods, vol. A237, nos. 1, 2, pp. 203-206, June 15, 1985.

[9] I. Kimel, L. R. Elias, and G. Ramian, "The UCSB two-stage FEL experiment," Nucl. Instrum. Methods, vol. A250, nos. 1, 2, pp. 320327, Sept. 1, 1986.

[10] D. Arnush, H. Boehmer, M. Z. Caponi, and C. C. Shih, "Design of a high power CW free electron maser," Int. J. Electron., vol. 53, no. 6. pp. 605-616. Dec. 1982.

[11] J. H. Booske, A. Serbeto, T. M. Antonsen, Jr., and B. Levush, "Nonlinear analyses for optimized short-period-wiggler free electron laser oscillators," J. Appl. Phys., vol. 65, no. 4, pp. 1453-1459, Feb. 15, 1989.

[12] J. H. Booske et al. " "Free electron laser with small period wiggler and sheet electron beam: A study of the feasibility of operation at 300 $\mathrm{GHz}$ with I MW CW output power." Nucl. Instrum. Methods, vol. A285, nos. 1, 2, pp. 92-96, Dec. 10, 1989.

[13] A. Serbeto, B. Levush, and T. M. Antonsen Jr., "Efficiency optimization for free-electron laser oscillators," Phys. Fluids $B$. vol. 1 . no. 2, pp. 435-439, Feb. 1989. 
[14] T. M. Antonsen Jr. and B. Levush, "Mode competition and suppression in free electron laser oscillators," Phys. Fluids B, vol. 1, no. 5, pp. 1097-1108, May 1989.

[15] T. M. Antonsen Jr. and B. Levush, "Mode competition and control in free-electron-laser oscillators," Phys. Rev. Lett., vol. 62, no. 13 , pp. 1488-1491, March 27, 1989.

[16] B. Levush and T. M. Antonsen Jr., "Regions of stability of FEL oscillators,"' Nucl. Instr. Meth. Phys. Res., vol. A272, nos. 1, 2, pp 375-379, Oct. 1988.

[17] T. M. Antonsen Jr. and P. Latham, "Linear theory of a sheet beam free electron laser," Phys. Fluids, vol. 31, no. 11, pp. 3379-3386, Nov. 1988.

[18] R. J. Harvey and F. A. Dolezal, "Stable single-mode operation of $30 \mathrm{GHz}$ free-electron laser,' Appl. Phys. Lett., vol. 53, no. 13, pp. $1150-1152$, Sept. 26, 1988.

[19] R. J. Harvey, Hughes Research Labs., private communications, 1989.

[20] T. M. Antonsen Jr. and B. Levush, "Spectral characteristics of a free electron laser with time dependent beam energy," Phys. Fluids $B$, to be published.

[2i] G. Ramian, J. Hu, S. G. Evangelides, T. S. Chu, B. G. Danly, R. J. Temkin, and T. G. Sollner, "Direct spectral measurements of the UCSB FEL," in the 11th Int. Conf. on Free Electron Lasers Conference Dig. New York: IEEE, 1989, pp. 142-143.

[22] S. W. Bidwell et al., "Designs and experiments for high average power FELs using sheet electron beams and short period wigglers," Bull. Amer. Phys. Soc., vol. 34, no. 9, p. 1984, Oct. 1989.

[23] T. J. Orzechowski et al., "High efficiency extraction of microwave radiation from a tapered-wiggler free-electron laser," Phys. Rev. Lett., vol. 57, no. 17, pp. 2172-2175, Oct. 27, 1986.

[24] K. E. Kreischer, T. L. Grimm, A. W. Mobius, and R. J. Temkin, "The design of megawatt gyrotrons for the Compact Ignition Tokamak," in 13th Int. Conf. Infrared and Millimeter Waves Conf. Dig. SPIE vol. 1039. Bellingham, WA: SPIE, 1988, pp. 179-180.

[25] A. T. Lin, N. C. Luhmann Jr., D. B. McDermott, and K. R. Chu, "Cyclotron autoresonant maser (CARM) EC heating source for high field tokamaks," in 12th Int. Conf. Infrared and Millimeter Waves Conf. Dig., IEEE Cat. No. 87CH2490-1. New York: IEEE, 1987, pp. 324-325.

Q. S. Wang, A. T. Lin, N. C. Luhmann Jr., D. B. McDermott, and K. R. Chu, "CARM EC heating source for high field tokamaks," in 13th Int. Conf. Infrared and Millimeter Waves Conf. Dig., SPIE vol. 1039. Bellingham, WA: SPIE, 1988, pp. 320-321.

[26] J. D. Jackson, Classical Electrodynamics. New York: Wiley, 1975.

[27] E. M. Marshall, P. M. Phillips, and J. E. Walsh, "Planar orotron experiments in the millimeter wavelength band," IEEE Trans. Plasma Sci., vol. 16, no. 2, pp. 199-205, Apr. 1988.

[28] E. T. Scharlemann, "Wiggle plane focusing in linear wigglers," $J$. Appl. Phys., vol. 58, no. 6, pp. 2154-2161, Sept. 15, 1985.

[29] H. P. Freund, H. Bluem, and C. L. Chang, "Three-dimensional nonlinear analysis of free-electron-laser amplifiers with planar wig glers,"' Phys. Rev. A, vol. 36, no. 5, pp. 2182-2198, Sept. 1, 1987.

[30] D. J. Radack, J. H. Booske, Y. Carmel, and W. W. Destler, "Wiggler focused relativistic sheet beam propagation in a planar free-electron laser configuration," Appl. Phys. Lett., vol. 55, no. 20, pp. 2069-2071, Nov. 13, 1989.

[31] R. Miller, Stanford Linear Accelerator Ctr., private communications, 1988.

[32] G. Miram, Varian, private communications, 1988.

[33] J. R. Pierce, Theory and Design of Electron Beams. Princeton, NJ: Van Nostrand, 1954, chap. 10.

[34] J. D. Lawson, The Physics of Charged Particle Beams. Oxford: Clarendon, 1977, chaps. 2-4.

[35] K. Amboss, "The analysis of dense electron beams," in Advances in Electronics and Electron Physics, vol. 26, L. Marton, Ed. New York: Academic, 1969, pp. 1-170.

[36] C. Andelfinger, W. Dommaschk, and W. Ott, "Bright high current $2 \mathrm{MeV}$ electron beam tube working in a poor vacuum," in 5 th Int. Symp. on Discharges and Electrical Insulation in Vacuum. Pozn'an, Poland: Politechnika Pozna'nska, 1972, pp. 383-387.

[37] P. R. Garabedian, "Stability of Cauchy's problem in space for analytic system of arbitrary type," J. Math. Mech., vol. 9, no. 6, pp 905-914, June 1960.

[38] K. J. Harker, "Determination of electrode shapes for axially symmetric electron guns," J. Appl. Phys., vol. 31, no. 12, pp. $2165-$ 2170 , Dec. 1960

[39] J. Fink, H. B. Schilling, and V. Schumacher, "Development and investigation of relativistic electron beams with finite energy spread and improved emittance," J. Appl. Phys., vol. 51, no. 6, pp. 2295-3000, June 1980.

[40] D. A. Kirkpatrick, R. E. Shefer, and G. Bekefi, "High brightness electrostatically focused field emission electron gun for free electron laser applications," J. Appl. Phys., vol. 57, no. 11, pp. 5011-5016, June 1, 1985.

[41] W. B. Herrmannsfeldt, "Electron Trajectory Program," Rep. SLAC226, Stanford Linear Accelerator Ctr., Nov. 1979.

[42] J. M. Baird and W. Lawson, "Magnetron injection gun (MIG) design for gyrotron applications,"' Int. J. Electron., vol. 61, no. 6, pp. 953967, Dec. 1986

[43] J. M. Finn, T. M. Antonsen Jr., and W. M. Manheimer, "Spacecharge-limited and temperature-limited electron flow in the vicinity of edges and conical points," IEEE Trans. Plasma Sci., vol. 16, no. 2, pp. 281-289, Apr. 1988.

[44] R. True, "Emittance and the design of beam formation, transport, and collection systems in periodically focused TWT's," IEEE Trans. Electron Devices, vol. ED-34, no. 2, pp. 473-485, Feb. 1987.

[45] W. Neugebauer and T. G. Mihran, "A ten-stage electrostatic depressed collector for improving klystron efficiency," IEEE Trans. Electron Devices, vol. ED-19, no. 1, pp. 111-121, Jan. 1972.

[46] H. G. Kosmahl, "Modern multistage depressed collectors-A review," Proc. IEEE, vol. 70, no. 11, pp. 1325-1334, Nov. 1982.

[47] M. L. Sundquist, J. A. Adney, S. J. Dehais, D. B. Cline, D. J. Larson, D. R. Anderson, and F. E. Mills, "Operation of a $3 \mathrm{MeV}$ ampere intensity dc electron recirculation system,"' in Proc. 1987 IEEE Particle Accelerator Conf., vol. 1. New York: IEEE, 1987, pp. 316-318.

[48] M. E. Read, A. J. Dudas, W. G. Lawson, and A. Singh, "Depressed collector for high power gyrotrons," IEEE Trans. Electron Devices, to be published.

[49] S. Meissel, Hipotronics Corp., private communications, 1989.

[50] T. C. Christiansen et al., "Development of a laced electromagnetic wiggler," IEEE Trans. Plasma Sci., vol. 16, pp. 1094-1097, Apr. 1988

[51] A. Friedman, Science Applications Int. Corp., private communications, 1989.

[52] R. L. Sheffield et al., "Workshop results on small-period wiggler designs," in Proc. 11th Int. Conf. on Free Electron Lasers. New York: IEEE, 1989, pp. 100-103.

[53] K. Batchelor et al., "A microwiggler free-electron laser at the Brookhaven Accelerator Test Facility," in Proc. 1/th Int. Conf. on Free Electron Lasers. New York: IEEE, 1989, pp. 116-117.

[54] J. D. Lee, Ed., "TIBER II/ETR final design report: Vol. 2, 3.0 Engineering," Lawrence Livermore Nat. Lab. Rep. UCID-21150, Vol 2, pp. 3.2-1-3.2-38, Sept. 1987.

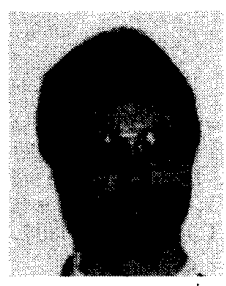

John H. Booske (S'82-M'89) received the Ph.D. degree in nuclear engineering from the University of Michigan, Ann Arbor, in 1985. Topics of graduate research included high-power $\mathrm{CO}_{2}$ lasers and electron-cyclotron resonant heating and cyclotron emissions from magnetically confined hot electron plasmas.

In 1985 he began work in the Laboratory for Plasma Research, University of Maryland, College Park, as a Research Associate, studying hot ion microinstabilities in magnetic mirror plasmas. He subsequently joined the Research and Graduate faculties at the University of Maryland, with responsibility for research in millimeter-wave free electron lasers. He is currently a faculty member in the Department of Electrical and Computer Engineering, University of Wisconsin, Madison. His research interests include free electron and plasma radiation sources, plasma heating and wave interactions, and millimeter-wave/dielectric interactions. 


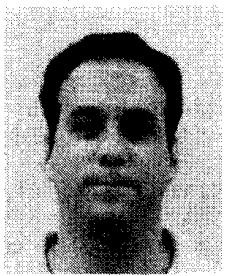

Daniel J. Radack received the B.S. degree in 1983, the M.S. degree in 1985, and the Ph.D. de gree in 1989, all in electrical engineering and from the University of Maryland, College Park. His $\mathrm{Ph}$.D. dissertation concerned the design of a novel short-period wiggler electromagnet for a freeelectron laser, as well as a demonstration of stable sheet electron-beam propagation through the wiggler.

From 1984 to 1985 he was a Graduate Research Fellow in the Semiconductor Electronics Division of the National Bureau of Standards, working on the accurate measurement of propagation delay in integrated circuits. In 1986 he joined the division as a Research Scientist. In 1987 he began working for the Charged Particle Beam Group in the Laboratory for Plasma Research at the University of Maryland. His work involved research into the solution of the basic problems encountered in the design of a high-power, millimeterwavelength free electron laser. He became a member of the research faculty there in 1990. His current interests include FEL's, gyrotrons, accelerators, and novel magnetic devices.

Dr. Radack is a member of Eta Kappa Nu and Tau Beta Pi.

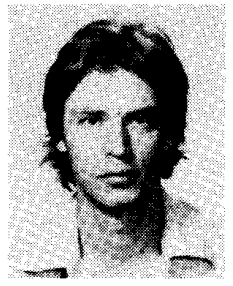

Thomas M. Antonsen, Jr. (M'87) was born in Hackensack, NJ, on December 7,1950 . He re ceived the B.S., M.S., and Ph.D. degrees in electrical engineering from Cornell University, Ithaca, NY, in 1973, 1976, and 1977, respectively.

After graduating from Cornell, he held a postdoctoral appointment at the Naval Research Laboratory, Washington, DC. From 1977 to 1980 he was a Research Scientist at the Massachusetts Institute of Technology, Cambridge. In 1980 he moved to the University of Maryland, College Park, where he became Professor in the Department of Electrical Engineering and Physics in 1989. His current research interests include highpower radiation from free-electron lasers and gyrotrons, magnetically confined fusion plasmas, and nonlinear dynamics.

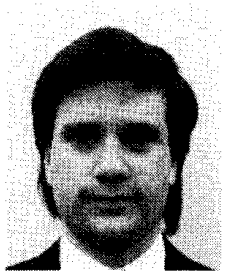

Steven W. Bidwell received the Ph.D. degree in nuclear engineering from the University of Michigan, Ann Arbor, in 1989. His graduate work concentrated on the interaction of intense relativistic electron beams with flames and gases.

Following graduation, he accepted a Research Associate position at the University of Maryland's Laboratory for Plasma Research, College Park, where his pursuits include experimental research in free-electron laser physics and the development of FEL sources of millimeter-wave radiation for heating magnetic fusion plasmas.

Dr. Bidwell is a member of the American Physical Society and the $\mathrm{Na}$ tional Society of Professional Engineers.

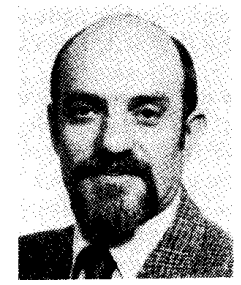

Yuval Carmel (S'66-M'74) was born in Israel in 1942. He received the B.Sc.(EE) and M.Sc.(EE) degrees from the Technion, Israel Institute of Technology, in 1966 and 1971, respectively, and the Ph.D.(EE) degree from Cornell University, Ithaca, NY, in 1974

He was with the Government of Israel, the $\mathrm{Na}$ val Research Laboratory, and is currently with the University of Maryland, College Park. His research interests include electromagnetic radiation from intense electron beams, free electron lasers, advanced concepts in millimeter-wave tubes, gyrotrons, and backward wave oscillators.
William W. Destler (M'84), photograph and biography not available at the time of publication.

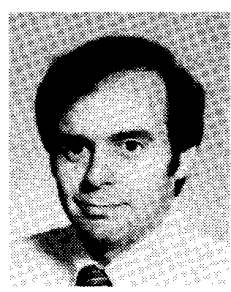

Henry P. Freund was born in New York City on May 23, 1949. He received the B.S. degree in physics from the Rensselaer Polytechnic Institute. Troy. NY, in 1971 , and the $\mathrm{Ph} . \mathrm{D}$. degree in physics from the University of Maryland, College Park, in 1976.

After graduation, he spent a year as a Research Fellow at the Institute for Physical Sciences and Technology, University of Maryland, College Park, and two years as a National Research Council-Naval Research Laboratory Postdoctoral Research Associate. His current research is devoted primarily to coherent radiation sources at the Naval Research Laboratory. At present, he is with the Plasma Physics Division, Science Applications International Corporation, McLean, VA.

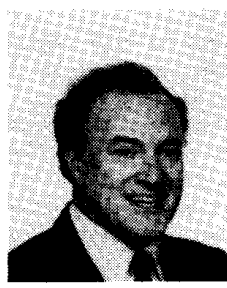

Victor L. Granatstein (S'59-M'64-SM'86) received the $\mathrm{Ph} . \mathrm{D}$. degree in 1963 from Columbia University, New York City, in electrical engineering and plasma physics.

After a year of postdoctoral work at Columbia University, he was a member of the Technical Staff of Bell Telephone Laboratories from 19641972. During 1969-1970 he was a Visiting Senio Lecturer at the Hebrew University of Jenusalem. In 1972 he joined the Naval Research Laboratory as a Research Physicist and from 1978-1983 served as Head of the High-Power Electromagnetic Radiation Branch. In August 1983 he became a Professor in the Electrical Engineering Department at the University of Maryland, College Park, and also serves as a Consultant to the Naval Research Laboratory, the Science Applications International Corporation, the Jet Propulsion Laboratory, and the State of Maryland (Department of Natural Resources). He is presently leading experimental and theoretical studies of electromagnetic radiation from relativistic electron beams, advanced concepts in millimeter-wave tubes, free electron lasers, and gyrotron amplifiers. He is Associate Editor of the International Journal of Electronics and has been a Guest Editor of the IEEE Transactions on Microwave Theory and Techniques and the IEEE Journal OF QUANTUM Electronics. He has been a reviewer for the NSF, AFOSR, DOE DNA, ONR, and ARO. He has co-authored more than 100 research papers in regular journals, and holds a number of patents on active and passive microwave devices. Since 1988 he has been Director of the Laboratory for Plasma Research at the University of Maryland.

Professor Granatstein is a Fellow of the American Physical Society.

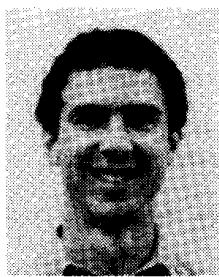

Peter E. Latham (M'87) received the B.A. de gree from the University of California at San Diego in 1977. He then went on to the University of California at Berkeley, where he received the M.A. degree in physics in 1979, and the Ph.D degree in physics in 1986. Currently he is working on high-power microwave devices, including gyrotrons and free electron lasers, at the University of Maryland, College Park.

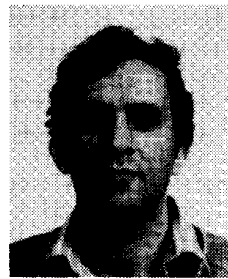

Baruch Levush (M'88) was born in Yackutsk, USSR, on March 23, 1950. He received the M.S. degree in physics from Latvian State University, Riga, Latvia, USSR, in 1972, and the Ph.D. de gree in physics from Tel-Aviv University, TelAviv, Israel, in 1981 .

After graduating from Tel-Aviv University, he received a Dr. Chaim Weizman Postdoctoral Fellowship and stayed for two and a half years at the University of Maryland, College Park, as a Post doctoral Fellow. From 1984 to 1985 he was a Re 
search Scientist with the Rafael Research Laboratory, Israel. In 1985 he joined the research staff of the Laboratory for Plasma Research, University of Maryland, where in 1988 he became an Associate Research Scientist. His current research interests include the physics of coherent radiation sources such as free electron lasers, gyrotrons and BWO's, and high current accelerators.

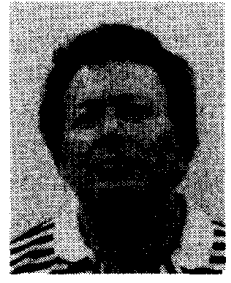

Isaak D. Mayergoyz (SM'83-F'88) was born on April 10, 1941 in Kiev, USSR. He received the $\mathrm{Ph} . \mathrm{D}$. degree in electrical engineering from the Institute of Cybernetics of the Ukranian Academy of Sciences (Kiev) in 1968.

He worked at the Institute of Cybernetics from 1966 until 1979 as a Senior Scientist. In 1980 he emigrated to the United States. Since 1981 he has been a Professor of Electrical Engineering at the University of Maryland, College Park. His research interests are in the areas of electromagnet- ics, applied superconductivity, numerical modeling of semiconductor devices, power engineering, and applied mathematics.

Dr. Mayergoyz is a Research Fellow of GE Corporate Research and Development (1988), a member of the Electromagnetics Society, and a recipient of the Outstanding Teaching Award of the College of Engineering, University of Maryland.

A. Serbeto, photograph and biography not available at the time of publication. 